\title{
VARIATIONAL PRINCIPLES IN THE THEORY OF FINITE PLASTIC DEFORMATIONS*
}

\author{
BY \\ ARIS PHILLIPS \\ Stanford University
}

1. Introduction. In the present paper we give the variational principles of the theory of finite plastic deformations taking into account strain-hardening. Throughout the paper only Lagrangian coordinates will be used. This work is based on a paper of $\mathbf{R}$. Kappus, ${ }^{1}$ where a very clear exposition of the theory of finite elastic deformations is given.

The strain components are

$$
g_{p q}=u_{a, p}+u_{p, a}+u_{i, p} u_{i, a},
$$

where $u_{p}$ is the displacement and a subscript after a comma denotes differentiation with respect to the corresponding coordinate. The geometrical significance of these strain components is the following. Let us consider the system $\left(O, e_{i}^{(1)}, e_{i}^{(2)}, e_{i}^{(3)}\right)$ of the particle $O$ of the body and the three unit vectors $e_{i}^{(1)}, e_{i}^{(2)}, e_{i}^{(3)}$ which are parallel to the axes of the fixed coordinate system. The deformation brings $O$ to $O_{1}$, and $e_{i}^{(1)}, e_{i}^{(2)}, e_{i}^{(3)}$ to $g_{i}^{(1)}, g_{i}^{(2)}, g_{i}^{(3)}$, respectively.

The strain components $g_{p q}$ are given by the formulas

$$
g_{p q}=g_{i}^{(p)} g_{i}^{(a)}-\delta_{p a},
$$

where $\delta_{p q}$ is the Kronecker delta. They are components of a tensor.

Let us consider now the cubic element built on the vectors $e_{i}^{(1)}, e_{i}^{(2)}, e_{i}^{(3)}$. After deformation has taken place it became a parallelopiped built on the three vectors $g_{i}^{(1)}, g_{i}^{(2)}, g_{i}^{(3)}$. The forces on the faces of this parallelopiped are $\tau_{i}^{(1)}, \tau_{i}^{(2)}, \tau_{i}^{(3)}$.

Let us resolve them as follows:

$$
\tau_{i}^{(p)}=\tau_{p e} g_{i}^{(\alpha)} .
$$

The components $\tau_{p a}$ are called the stress components. They are the components of a tensor and they fulfill the conditions

$$
\tau_{p q}=\tau_{a p}
$$

The equations of equilibrium are

$$
\left(\tau_{i q}+\tau_{p q} u_{i, p}\right)_{, a}+F_{i}=0
$$

and the boundary conditions are

$$
\left(\tau_{i q}+\tau_{p q} u_{i, p}\right) \nu_{q}=F_{i}^{\prime},
$$

where $\nu_{q}$ are the direction cosines of the exterior normal.

The stress-strain relations of the theory of finite elastic deformations are taken in the form

*Received May 10, 1948. The results presented in this paper were obtained in the course of research conducted under contract, sponsored by the Office of Naval Research.

${ }^{1}$ R. Kappus, Z. angew. Math. Mech. 19, 271 (1939). 


$$
\tau_{p q}=G\left(g_{p q}-\frac{1}{3} g_{i i} \delta_{p q}\right)+K \frac{1}{3} g_{i i} \delta_{p q} .
$$

By putting

$$
\begin{array}{cc}
\tau_{p q}^{*}=\tau_{p q}-\frac{1}{3} \tau_{i i} \delta_{p q}, & g_{p q}^{*}=g_{p q}-\frac{1}{3} g_{i i} \delta_{p q}, \\
T^{*}=\left(\tau_{p q}^{*} \tau_{p q}^{*}\right)^{1 / 2}, & D^{*}=\left(g_{p q}^{*} g_{p q}^{*}\right)^{1 / 2},
\end{array}
$$

we get the two equations

$$
\tau_{i i} / g_{i i}=k
$$

$$
T^{*}=G D^{*} .
$$

The theory of finite plastic deformations assumes that Eq. (9) is valid again but that Eq. (10) will be replaced by

$$
T^{*}=f\left(D^{*}\right)
$$

i.e. $T^{*}$ is a function of $D^{*}$.

Thus Eq. (7) becomes

$$
\tau_{p q}=\frac{f\left(D^{*}\right)}{D^{*}} g_{p q}^{*}+K \frac{1}{3} g_{i i} \delta_{p q} .
$$

Solving Eq. (11) for $D^{*}$ we get

$$
D^{*}=\phi\left(T^{*}\right)
$$

and

$$
g_{p q}=\frac{\phi\left(T^{*}\right)}{T^{*}} \tau_{p q}^{*}+\frac{1}{K} \frac{1}{3} \tau_{i i} \delta_{p q} .
$$

2. The variational principle for the virtual displacements. Let us consider the function

$$
A_{0}=\int_{0}^{D^{*}} f\left(D^{*}\right) d D^{*}+\frac{3}{2} K g^{2},
$$

where $g=g_{i i} / 3$. We find

$$
\begin{aligned}
\frac{\partial A_{0}}{\partial g_{p q}} & =f\left(D^{*}\right) \frac{\partial D^{*}}{\partial g_{p q}}+3 K g \frac{\partial g}{\partial g_{p q}} \\
& =\frac{f\left(D^{*}\right)}{D^{*}} g_{p q}^{*}+K g \delta_{p q}=\tau_{p q} .
\end{aligned}
$$

We consider now a body in equilibrium under the action of external forces. We denote by $\tau_{p a}$ the actual stress components, by $u_{i}$ the components of the actual displacements and by $g_{p q}$ the actual strain components, due to the loads.

We compare the actual value of the expression

$$
P=\int_{V} A_{0} d V-2 \int_{V} F_{i} u_{i} d V-2 \int_{S} F_{i}^{\prime} u_{i} d S
$$

with the value which this expression would have if $u_{i}$ and $g_{p q}$ were submitted to small variations $\delta u_{i}, \delta g_{p q}$ while the stress components and the external forces remained un- 
changed. These variations are not entirely free; the new strain components $g_{p q}+\delta g_{p q}$ and displacements $u_{i}+\delta u_{i}$ must fulfill the following conditions:

a) the condition of continuity,

b) the geometrical boundary condition,

c) the equations of equilibrium

$$
\left\{\tau_{i q}+\tau_{p q}\left(u_{i}+\delta u_{i}\right)_{, p}\right\}_{, a}+F_{i}=0,
$$

d) the statical boundary conditions

$$
\left\{\tau_{i q}+\tau_{p q}\left(u_{i}+\delta u_{i}\right)_{, p}\right\} \nu_{q}=F_{i}^{\prime} .
$$

We shall prove that the actual value of $P$ is a minimum. Let us write $A=\int_{V} A_{0} d V$. We get

$$
\delta A=\int_{V} \delta A_{0} d V=\int_{V} \frac{\partial A_{0}}{\partial g_{p q}} \delta g_{p q} d V=\int_{V} \tau_{p q} \delta g_{p q} d V
$$

Using the Eqs. (1) we obtain

$$
\delta A=\int_{V}\left[\tau_{p q}\left(\delta u_{p, q}+\delta u_{q, p}+u_{i, p} \delta u_{i, q}+u_{i, Q} \delta u_{i, p}\right)\right] d V .
$$

Integrating by parts and noting Eq. (4), we find that Eq. (20) becomes

$$
\begin{aligned}
\delta A= & -\int_{V}\left[\delta u_{p} \tau_{p q, a}+\delta u_{q} \tau_{p q, p}+\delta u_{i}\left(\tau_{p q} u_{i, p}\right)_{, a}\right. \\
& \left.+u_{i}\left(\tau_{p q} \delta u_{i, q}\right)_{, p}+\delta u_{i}\left(\tau_{p q} u_{i, a}\right)_{, p}+u_{i}\left(\tau_{p q} \delta u_{i, p}\right)_{, q}\right] d V \\
& +\int_{s}\left[\delta u_{p} \tau_{p q} \nu_{q}+\delta u_{q} \tau_{p q} \nu_{p}+\delta u_{i}\left(\tau_{p q} u_{i, p}\right) \nu_{q}\right. \\
& +u_{i}\left(\tau_{p q} \delta u_{i, q}\right) \nu_{p}+\delta u_{i}\left(\tau_{p q} u_{i, q}\right) \nu_{p} \\
& \left.+u_{i}\left(\tau_{p q} \delta u_{i, p}\right) \nu_{q}\right] d S .
\end{aligned}
$$

Using the Eqs. (5), (6) and (17), (18) we get

$$
\delta A=2 \int_{V} F_{i} \delta u_{i} d V+2 \int_{S} F_{i}^{\prime} \delta u_{i} d S,
$$

and because $F_{i}, F_{i}^{\prime}$ remain constant during the variations,

$$
\delta\left[\int_{V} A_{0} d V-2 \int_{V} F_{i} u_{i} d V-2 \int_{S} F_{i}^{\prime} u_{i} d S\right]=0
$$

or

$$
\delta P=0 .
$$

This means that $P$ is an extremum. We have to prove now that $P$ is a minimum. We have

$$
\delta^{2} P=\delta^{2} \int_{V} A_{0} d V=\int_{V} \delta^{2} A_{0} d V
$$


But

$$
\delta^{2} A_{0}=\frac{d f\left(D^{*}\right)}{d D^{*}}\left(\delta D^{*}\right)^{2}+f\left(D^{*}\right) \delta^{2} D^{*} .
$$

The first product of the right member of this equation is positive because for all materials $d f\left(D^{*}\right) / d D^{*}>0$. Let us examine the second product. We have

$$
\delta^{2} D^{*}=\frac{\partial^{2} D^{*}}{\partial g_{p q}^{*} \partial g_{r s}^{*}} \delta g_{p q}^{*} \delta g_{r s}^{*} .
$$

But

$$
\frac{\partial^{2} D^{*}}{\partial g_{p q}^{*} \partial g_{r s}^{*}}=\frac{D^{*} \delta_{p a r s}^{2}-g_{p q}^{*} g_{r s}^{*}}{D^{* 3}}
$$

where

$$
\delta_{p q r s}=\left\{\begin{array}{lll}
1 & \text { for } & p q=r s \\
0 & \text { for } & p q \neq r s .
\end{array}\right.
$$

Hence

$$
\begin{aligned}
\delta^{2} D^{*} & =\frac{D^{*^{2}}\left(\delta g_{i j}^{*} \delta g_{i j}^{*}\right)-\left(g_{i j}^{*} \delta g_{i j}^{*}\right)^{2}}{D^{*^{3}}} \\
& =\frac{1}{D^{*^{3}}}\left[\left(g_{p q}^{*} \delta g_{r_{s}}^{*}-g_{r_{s}}^{*} \delta g_{p a}^{*}\right)\left(g_{p q}^{*} \delta g_{r_{s}}^{*}-g_{r_{s}}^{*} \delta g_{p q}^{*}\right)\right]>0 .
\end{aligned}
$$

Therefore $\delta^{2} A>0$ and $\delta^{2} P>0$.

3. The variational principle for the virtual stress variations. Let us consider the function

$$
B_{0}=\int_{0}^{T^{*}} \phi\left(T^{*}\right) d T^{*}+\frac{3}{2} \frac{\tau^{2}}{K}
$$

where $\tau=\tau_{i i} / 3$. We have

$$
\begin{aligned}
\frac{\partial B_{0}}{\partial \tau_{p q}} & =\phi\left(T^{*}\right) \frac{\partial T^{*}}{\partial \tau_{p q}}+\frac{3}{K} \tau \frac{\partial \tau}{\partial \tau_{p q}} \\
& =\frac{\phi\left(T^{*}\right)}{T^{*}}\left(\tau_{p q}-\tau \delta_{p q}\right)+\frac{\tau}{K} \delta_{p q}=g_{p q} .
\end{aligned}
$$

Let us consider again a body in equilibrium under the action of external forces. We consider the expression

$$
Q=\int_{V} B_{0} d V-2 \int_{S_{0}} F_{i}^{\prime} u_{i} d S
$$

in which the second integral is extended over the part of the surface of the body where the boundary conditions are such that the external forces $F_{i}^{\prime}$ are not given. We compare the actual value of $Q$ with the value which $Q$ would have if the stress components 
and the surface forces were submitted to small variations, while the strain components, the displacements, and the body forces remained unchanged. The only conditions which the variations must fulfill are that the new stress components and boundary forces must satisfy the equilibrium equations and the boundary conditions. We shall prove that the actual value of $Q$ is a minimum.

Let us write

We get

$$
B=\int_{V} B_{0} d V
$$

$$
\delta B=\int_{V} \delta B_{0} d V=\int_{V} \frac{\partial B_{0}}{\partial \tau_{p q}} d V=\int_{V} g_{p q} \delta \tau_{p q} d V .
$$

Using the Eqs. (1) we find that

$$
\delta B=\int_{V}\left[\delta \tau_{p q}\left(u_{p, a}+u_{a, p}+u_{i, p} u_{i, q}\right)\right] d V .
$$

Integrating by parts we obtain

$$
\begin{aligned}
\delta B= & -\int_{V}\left[u_{p} \delta \tau_{p q, q}+u_{q} \delta \tau_{p q, p}+u_{i}\left(\delta \tau_{p q} u_{i, q}\right)_{, p}\right. \\
& \left.+u_{i}\left(\delta \tau_{p q} u_{i, p}\right)_{, q}\right] d V+\int_{S}\left[u_{p} \delta \tau_{p q} \nu_{q}\right. \\
& \left.+u_{q} \delta \tau_{p q} \nu_{p}+u_{i}\left(\delta \tau_{p q} u_{i, q}\right) \nu_{p}+u_{i}\left(\delta \tau_{p q} u_{i, p}\right) \nu_{q}\right] d S
\end{aligned}
$$

in which the second integral is valid for the whole boundary. The stress variations have been selected such that the equations of equilibrium are always satisfied. Hence the first integral vanishes.

In the part of the boundary where the external forces are given the second integral vanishes because the stress variations have been selected so that the boundary equations are satisfied. The second integral is different from zero in those parts of the boundary only where the external forces are not given. Then we have

$$
\delta B=2 \int_{S_{\circ}} u_{i} \delta F_{i}^{\prime} d S,
$$

where $S_{0}$ means the above-mentioned part of the boundary. As $u_{i}$ remain constant during the variation, we get

and

$$
\delta\left[\int_{V} B_{0} d V-2 \int_{S_{0}} u_{i} F_{i}^{\prime} d S\right]=0
$$

$$
\delta Q=0 .
$$

Hence $Q$ is an extremum. The proof that $Q$ is a minimum is similar to the proof for the minimum property of $P$.

The variational principles of the theory of infinitely small plastic deformations are special cases of the two principles given above. These special cases have been treated by L. M. Kachanov ${ }^{2}$ and by the author ${ }^{3}$ independently.

${ }^{2}$ L. M. Kachanov, Prikl. Mat. Mekh. 6, 187-196 (1942).

${ }^{3}$ A. Philippidis, Spannungen und Verformungen im überelastischen Bereich, Münich, 1944. Unpublished Report. 